\title{
Foundations of not necessarily rational Negative Imaginary systems theory: Relations between classes of Negative Imaginary and Positive Real systems
}

DOI:

10.1109/TAC.2015.2497461

\section{Document Version}

Accepted author manuscript

Link to publication record in Manchester Research Explorer

Citation for published version (APA):

Ferrante, A., Lanzon, A., \& Ntogramatzidis, L. (2015). Foundations of not necessarily rational Negative Imaginary systems theory: Relations between classes of Negative Imaginary and Positive Real systems. I E E E Transactions on Automatic Control. https://doi.org/10.1109/TAC.2015.2497461

Published in:

I E E E Transactions on Automatic Control

\section{Citing this paper}

Please note that where the full-text provided on Manchester Research Explorer is the Author Accepted Manuscript or Proof version this may differ from the final Published version. If citing, it is advised that you check and use the publisher's definitive version.

\section{General rights}

Copyright and moral rights for the publications made accessible in the Research Explorer are retained by the authors and/or other copyright owners and it is a condition of accessing publications that users recognise and abide by the legal requirements associated with these rights.

\section{Takedown policy}

If you believe that this document breaches copyright please refer to the University of Manchester's Takedown Procedures [http://man.ac.uk/04Y6Bo] or contact uml.scholarlycommunications@manchester.ac.uk providing relevant details, so we can investigate your claim.

\section{OPEN ACCESS}




\title{
Foundations of not necessarily rational Negative Imaginary systems theory: Relations between classes of Negative Imaginary and Positive Real systems
}

\author{
Augusto Ferrante, Alexander Lanzon, Lorenzo Ntogramatzidis
}

\begin{abstract}
In this paper we lay the foundations of a not necessarily Abstract-In this paper we lay the foundations of a not necessarily
rational negative imaginary systems theory and its relations with positive real systems theory. In analogy with the theory of positive real functions, in our general framework negative imaginary systems are defined in terms of a domain of analyticity of the transfer function and of a sign condition that must be satisfied in such domain. In this way, we do not require to restrict the attention to systems with a rational transfer function. In this work, we also define various grades of negative imaginary systems and aim to provide a unitary view of the different notions that have appeared so far in the literature within the framework of positive real and in the more recent theory of negative imaginary systems, and to show how these notions are characterized and linked to each other.
\end{abstract}

\section{INTRODUCTION}

The theory of positive real systems is one of the fundamental cornerstones of systems and control theory, and in particular of passivity theory. Given the extensive amount of contributions in this area, dating back from the early 1930s [1], it would be impossible to quote all of the relevant references. We consequently refer the readers to two important monographs [2], [3] for a summary of the historic and recent contributions on this problem. A promising recent new development has been the introduction of the notion of negative imaginary systems, see [4], [5], [6] and the references cited therein. With respect to positive realness, the definition of negative imaginary system imposes a weaker restriction on the relative degree of the transfer function and does not exclude all unstable zeros. Negative imaginary systems theory was found to be very suitable in a range of applications including modelling and control of undamped or lightly damped flexible structures with colocated position sensors and force actuators [7], [8], in nano-positioning control due to piezoelectric transducers and capacitive sensors (e.g. [9], [10], [11]) and in multiagent networked systems (e.g. [12], [13]). The notion of negative imaginary system specializes also to the important subclass of lossless negative imaginary systems [14].

In spite of the wealth of results that in just a few years have been presented and published on negative imaginary systems including extensions to infinite dimensional systems [15], Hamiltonian systems [16], descriptor systems [17] and mixtures of negative imaginary and small-gain properties [18] to mention only a few, so far [20] has been to the best of the authors' knowledge the only contribution which attempted to address the general case of a definition of negative imaginary system for not necessarily rational transfer functions. However, several aspects of the core theory of negative imaginary systems remained unexplored in [20]. For example, the notion of strictly negative imaginary system has never been defined in the

Augusto Ferrante is with the Dipartimento di Ingegneria dell'Informazione, Università di Padova, via Gradenigo, 6/B - 35131 Padova, Italy. E-mail: augusto@dei.unipd.it.

Alexander Lanzon is with the Control Systems Centre, School of Electrical and Electronic Engineering, University of Manchester, Sackville Street, Manchester M13 9PL, UK. E-mail: Alexander. Lanzon@manchester.ac.uk.

L. Ntogramatzidis is with the Department of Mathematics and Statistics, Curtin University, Perth, Australia. E-mail: L. Ntogramatzidis@curtin.edu.au.

Authors ordered alphabetically. Joint first authors. Partially supported by the Australian Research Council under the grant FT120100604. general case of a non-rational transfer function. This remaining gap will also be filled in this paper as it is essential in studying stability interconnections of negative imaginary systems.

Thus, the main contribution of this paper is to present a general and foundational perspective of the recent theory of negative imaginary systems, and their relation with the classical theory of positive real systems. As pointed out in [3], since the early studies in the 1960s, there has been a proliferation of definitions of various types of strictly positive real systems. Our aim is to follow the approach of [3] in the attempt of defining different notions of strictly negative imaginary system and establishing a parallel between these definitions and their positive real counterparts. The standard notion of strictly negative imaginary system introduced in the literature so far corresponds to only one of these definitions. We will define, examine and characterize other notions of strictly negative imaginary functions.

Notation. Given a matrix $A$, the symbol $A^{\top}$ denotes the transpose of $A$ and $A^{*}$ denotes the complex conjugate transpose of $A$. We denote by $\sigma(A)$ the set of singular values of the matrix $A$ and by $\underline{\sigma}(A)$ the smallest of such singular values. Recall that given a real rational function $G(s)$ and a simple pole $p \in \mathbb{C}$ of $G(s)$, we have a unique decomposition $G(s)=G_{1}(s)+A /(s-p)$, where $G_{1}(s)$ is a rational function which is analytic in an open set containing $p$ and the (non-zero) matrix $A$ is the residue corresponding to the pole $p$. If $p$ is a double pole of $G(s)$, we have the unique decomposition $G(s)=G_{1}(s)+A_{1} /(s-p)+A_{2} /(s-p)^{2}$, where the matrix $A_{1}$ is the residue corresponding to the pole $p$. In this case, by analogy, we define the (non-zero) matrix $A_{2}$ to be the quadratic residue corresponding to the pole $p$. If $G(s)$ has a pole at infinity, it can be uniquely decomposed as $G(s)=G_{1}(s)+P(s)$, where $G_{1}(s)$ is a rational proper function and $P(s)=\sum_{i=1}^{k} A_{i} s^{i}$ is a homogeneous polynomial in $s$. We refer to $A_{i}$ as the $i$-th coefficient in the expansion at infinity of $G(s)$. The usual notations of $\geq 0$ and $>0$ are used to denote positive semidefiniteness and positive definiteness of Hermitian matrices, respectively. Let $G: \mathbb{C} \longrightarrow \mathbb{C}^{m \times m}$ be analytic or harmonic in a certain region $\Omega$ of $\mathbb{C}$, then $G$ is said to have full normal rank if there exists $s \in \Omega$ such that $\operatorname{det}[G(s)] \neq 0$. Given complex matrices $S_{1}, S_{2}$ and complex vectors $y_{1}, y_{2}, u_{1}, u_{2}, \alpha, \beta$ of compatible dimension satisfying $\left[\begin{array}{c}y_{1} \\ \alpha\end{array}\right]=S_{1}\left[\begin{array}{l}u_{1} \\ \beta\end{array}\right]$ and $\left[\begin{array}{c}\beta \\ y_{2}\end{array}\right]=S_{2}\left[\begin{array}{c}\alpha \\ u_{2}\end{array}\right]$, let $S_{1} \star S_{2}$ denote the Redheffer star product which maps $\left[\begin{array}{l}u_{1} \\ u_{2}\end{array}\right]$ to $\left[\begin{array}{l}y_{1} \\ y_{2}\end{array}\right]$. Furthermore, $F_{l}\left(S_{1}, S_{2}^{(1,1)}\right)\left(\right.$ resp. $\left.F_{u}\left(S_{2}, S_{1}^{(2,2)}\right)\right)$ denote the lower (resp. upper) linear fractional transformation. Finally, let $[P, Q]$ denote the positive feedback interconnection between systems $P$ and $Q$.

\section{REVIEW OF POSITIVE REAL AND STRICTLY POSITIVE REAL} SYSTEMS

In this section, for the sake of completeness we briefly recall the most important notions and results of positive real systems.

Definition 1: The transfer function $F: \mathbb{C} \longrightarrow \mathbb{C}^{m \times m}$ is positive real (PR) if

- $F(s)$ is analytic in $\{s \in \mathbb{C}: \mathfrak{R e}\{s\}>0\}$;

- $F(s)$ is real when $s$ is real and positive;

- $F(s)+F(s)^{*} \geq 0$ for all $s \in\{s \in \mathbb{C}: \mathfrak{R e}\{s\}>0\}$. 
For real, rational systems, we have the following characterization of PR systems.

Lemma 1: Let $F: \mathbb{C} \longrightarrow \mathbb{C}^{m \times m}$ be a real, rational transfer function. Then, $F(s)$ is PR if and only if

- $F(s)$ has no poles in $\{s \in \mathbb{C}: \mathfrak{R e}\{s\}>0\}$;

- $F(i \omega)+F(i \omega)^{*} \geq 0$ for all $\omega \in \mathbb{R}$ such that $s=i \omega$ is not a pole of $F(s)$;

- if $i \omega_{0}$, with $\omega_{0} \in \mathbb{R} \cup\{\infty\}$, is a pole of $F(s)$, it is a simple pole with Hermitian and positive semidefinite residue (recall that if $\omega_{0}$ is finite, the residue is defined by $K_{0} \stackrel{\text { def }}{=} \lim _{s \rightarrow i \omega_{0}}\left(s-i \omega_{0}\right) F(s)$, while if $\omega_{0}=\infty$, the residue is defined by $K_{\infty} \stackrel{\text { def }}{=} \lim _{\omega \rightarrow \infty} \frac{F(i \omega)}{i \omega}$ ).

We now present our definitions of strictly positive real systems. We warn the reader that there is no consensus in the literature on this definition and many different definitions have been proposed for this concept that can be distinguished via several grades of strength, see e.g. [3], [21] and [22] where various definitions and the relationship between them are discussed. In this paper, we shall consider two grades of strength and we shall refer to them as strongly and weakly strictly positive realness. We must observe, though, that in many past works systems that we define "strongly strictly positive real" are simply addressed as "strictly positive real".

Definition 2: The transfer function $F: \mathbb{C} \longrightarrow \mathbb{C}^{m \times m}$ is strongly strictly positive real (SSPR) if for some $\varepsilon>0$, the transfer function $F(s-\varepsilon)$ is PR and $F(s)+F(-s)^{\top}$ has full normal rank.

The property of SSPR can be equivalently checked via a strict sign condition in the domain of analyticity.

Lemma 2: Let $F: \mathbb{C} \longrightarrow \mathbb{C}^{m \times m}$ be a real transfer function. Then, $F(s)$ is SSPR if and only if there exists $\varepsilon>0$ such that

(i) $F(s)$ is analytic in $\{s \in \mathbb{C}: \mathfrak{R e}\{s\}>-\varepsilon\}$;

(ii) $F(s)+F(s)^{*}>0$ for all $s \in\{s \in \mathbb{C}: \mathfrak{R e}\{s\}>-\varepsilon\}$.

The proof of this result can be carried out by adapting the proof of Lemma 4 in the sequel, and it is therefore omitted.

The following result, see [3, Theorem 2.47] and [21, Lemma 6.1], shows that in the case of real, rational functions the property of SSPR is equivalent to an analyticity condition and a sign condition restricted to the extended imaginary axis.

Theorem 1: Let $F: \mathbb{C} \longrightarrow \mathbb{C}^{m \times m}$ be a real, rational, proper transfer function. Then $F(s)$ is SSPR if and only if

1) $F(s)$ has all its poles in $\{s \in \mathbb{C}: \mathfrak{R e}\{s\}<0\}$;

2) $F(i \omega)+F(-i \omega)^{\top}>0$ for all $\omega \in \mathbb{R}$;

3) one of the three conditions is satisfied: ${ }^{1}$

- $F(\infty)+F(\infty)^{\top}>0$

- $F(\infty)+F(\infty)^{\top}=0$ and $\lim _{\omega \rightarrow \infty} \omega^{2}\left[F(i \omega)+F(-i \omega)^{\top}\right]>0$

- $F(\infty)+F(\infty)^{\top} \geq 0$ but not zero nor non-singular, and there exist $\sigma_{0}>0$ and $\delta>0$ such that

$$
\underline{\sigma}\left[\omega^{2}\left(F(i \omega)+F(-i \omega)^{\top}\right)\right] \geq \sigma_{0} \quad \forall|\omega| \geq \delta .
$$

Remark 1: Condition (1) has been a source of confusion and controversy in the literature for more than a decade. Indeed, the same condition was present in the second edition (published in 1996) of the book of Khalil (see Lemma 10.1); in the third edition [21] (published in 2002) this condition was changed with a new condition (see [21, Lemma 6.1]) that was easier to check. This new condition, however, has some inconsistencies as pointed out in [23] where the following further condition was derived that is more elegant and computationally easier to check:

$$
\lim _{|\omega| \rightarrow \infty} \omega^{2 \rho} \operatorname{det}\left(F(i \omega)+F(-i \omega)^{\top}\right)>0
$$

${ }^{1}$ We write this property as three separate conditions to elucidate all the possible situations that may occur. It is clear, however, that the third condition is the more general and encompasses the first and the second. where $\rho$ is the dimension of $\operatorname{ker}\left(F(\infty)+F(\infty)^{\top}\right)$. In [24] another condition was presented that hinges on a state-space realisation of $F(s)$. Here, we consider condition (1) for which we can establish a counterpart for negative-imaginary systems. Notice also that condition (1) is logically very simple and intuitive: it simply says that for $|\omega|$ sufficiently large, the spectral density $F(i \omega)+F(-i \omega)^{\top}$ is bounded from below by $\left(\sigma_{0} / \omega^{2}\right) I$ or, equivalently, that for $|\omega|$ sufficiently large, the spectral density $\omega^{2}\left[F(i \omega)+F(-i \omega)^{\top}\right]$ is bounded away from zero.

In some situations the concept of SSPR is too restrictive: indeed in the real, rational case where there are finitely many poles and zeros, it is useful to introduce the following weaker definition.

Definition 3: Let $F: \mathbb{C} \longrightarrow \mathbb{C}^{m \times m}$ be a real, rational, proper transfer function. Then, $F(s)$ is weakly strictly positive real (WSPR) if the first two properties of Theorem 1 hold.

Remark 2: If in the definition of SSPR we removed the full normal rank condition on $F(s)+F(-s)^{\top}$, we would have that functions such as $F(s)=\frac{1}{s+1}\left[\begin{array}{ll}1 & 1 \\ 1 & 1\end{array}\right]$ is SSPR so that a result like Theorem 1 would not hold. An example of a transfer function which is WSPR but not SSPR is the following:

$$
F(s)=\frac{s+3}{(s+1)(s+2)} .
$$

Indeed, 1) in Theorem 1 is satisfied. Moreover, given $\varepsilon>0$, a simple calculation gives

$$
F(i \omega-\varepsilon)+F(i \omega-\varepsilon)^{*}=2 \frac{6+6 \varepsilon^{2}-\varepsilon^{3}-\varepsilon\left(11+\omega^{2}\right)}{\left[\omega^{2}+(2-\varepsilon)^{2}\right]\left[\omega^{2}+(1-\varepsilon)^{2}\right]},
$$

which is strictly positive on the imaginary axis (i.e., when $\varepsilon=0$ ), so that 2) in Theorem 1 also holds. On the other hand, 3) in Theorem 1 is not satisfied. In fact, in this case $F(\infty)+F(\infty)^{\top}=0$, but $\lim _{\omega \rightarrow \infty} \omega^{2}\left[F(i \omega)+F^{\top}(-i \omega)\right]=\lim _{\omega \rightarrow \infty} \frac{12 \omega^{2}}{\left(\omega^{2}+4\right)\left(\omega^{2}+1\right)}=0$. This result is consistent with Definition 2. In fact, (2) shows that for any arbitrarily small $\varepsilon>0$, by taking a sufficiently large $\omega>0$, the numerator of $F(i \omega-\varepsilon)+F(i \omega-\varepsilon)^{*}$ can be rendered negative. In other words, $F(i \omega)+F(-i \omega)^{\top}$ is positive definite for all $\omega>0$, but no matter how small we choose $\varepsilon>0$, if $\omega>0$ is sufficiently large we can find $F(i \omega-\varepsilon)+F(i \omega-\varepsilon)^{*}<0$, and therefore $F(s-\varepsilon)$ is not PR for any $\varepsilon>0$.

The difference between SSPR and WSPR has also been discussed in [19], where however only the scalar case is considered.

\section{NegATIVE IMAGiNARY AND STRICTLY NEGATIVE IMAGINARY SYSTEMS}

We start this section by introducing the following standing assumption, that will be used throughout the rest of the paper.

Assumption 3.1: We henceforth restrict our attention to only symmetric transfer functions.

As discussed in [20], the case of symmetric transfer function is the most important and interesting one, because it encompasses both the scalar case, and the case of a transfer function of a reciprocal $m$-port electrical network. ${ }^{2}$ Moreover, to the best of the authors' knowledge, all the negative imaginary transfer functions considered or studied in the literature so far are symmetric (see e.g. the transfer functions from a force actuator to a corresponding collocated position sensor - for instance, a piezoelectric sensor - in a lightly damped or undamped structure), even though the real, rational definitions of negative imaginary systems in [4], [5], [6] allow for non-symmetric

${ }^{2}$ The only way to obtain a non-symmetric transfer function of an $\mathrm{m}$ port electrical network is to employ gyrators, whose physical implementation requires the use of active components. 
transfer functions. Assumption 3.1 is essential for, and underpins, the theory presented in this paper. How to capture the concept of a NI transfer function in the non-rational case without Assumption 3.1 is an open problem.

Definition 4: The real transfer function $G: \mathbb{C} \longrightarrow \mathbb{C}^{m \times m}$ is negative imaginary (NI) if

(i) $G(s)$ is analytic in $\{s \in \mathbb{C}: \mathfrak{R e}\{s\}>0\}$;

(ii) $i\left[G(s)-G(s)^{*}\right] \geq 0$ for all $s \in\{s \in \mathbb{C}: \mathfrak{R e}\{s\}>0, \mathfrak{I m}\{s\}>0\}$ (iii) $i\left[G(s)-G(s)^{*}\right]=0$ for all $s \in\{s \in \mathbb{C}: \mathfrak{R e}\{s\}>0, \mathfrak{I m}\{s\}=0\}$; (iv) $i\left[G(s)-G(s)^{*}\right] \leq 0$ for all $s \in\{s \in \mathbb{C}: \mathfrak{R e}\{s\}>0, \mathfrak{I m}\{s\}<0\}$.

The following result, which was proven in [20], provides a characterisation of real, rational NI systems in terms of a domain of analyticity and conditions referred to the imaginary axis.

Lemma 3: Let $G: \mathbb{C} \longrightarrow \mathbb{C}^{m \times m}$ be a real, rational, symmetric transfer function. Then $G(s)$ is NI if and only if

(i) $G(s)$ has no poles in $\{s \in \mathbb{C}: \mathfrak{R e}\{s\}>0\}$;

(ii) $i\left[G(i \omega)-G(i \omega)^{*}\right] \geq 0$ for all $\omega \in(0, \infty)$ except for the values of $\omega$ where $i \omega$ is a pole of $G(s)$;

(iii) if $s=i \omega_{0}$, with $\omega_{0} \in(0, \infty)$, is a pole of $G(s)$, then it is a simple pole and the corresponding residual matrix ${ }^{3} K_{0}=\lim _{s \rightarrow i \omega_{0}}(s-$ $\left.i \omega_{0}\right) i G(s)$ is Hermitian and positive semidefinite;

(iv) if $s=0$ is a pole of $G(s)$, then it is at most a double pole. Moreover, both its residual and its quadratic residual (when present) are positive semidefinite Hermitian matrices;

(v) if $s=\infty$ is a pole of $G(s)$, then it is at most a double pole. Moreover, both the coefficients in the expansion at infinity of $G(s)$ are negative semidefinite Hermitian matrices.

Remark 3: We observe that $\frac{1}{s}$ and $\frac{1}{s^{2}}$ are negative imaginary, whereas $-\frac{1}{s^{2}}$ is not. When there are poles on the imaginary axis, the Nyquist $D$-contour is indented infinitesimally to the right and hence the Nyquist plot changes phase rapidly at large magnitudes around the frequency of the pole(s) on the imaginary axis. From the complete Nyquist plot it is evident that $\frac{1}{s}$ and $\frac{1}{s^{2}}$ are negative imaginary, but $-\frac{1}{s^{2}}$ is not.

We recall the following important result, which established a relationship between PR and NI transfer functions, see [4], [5], [20].

Theorem 2: Let $G(s)$ be a real, rational, proper, symmetric negative imaginary transfer function matrix. Then $F(s) \stackrel{\text { def }}{=} s[G(s)-G(\infty)]$ is positive real. Conversely, let $F(s)$ be real, rational, symmetric positive real transfer function matrix. Then $G(s) \stackrel{\text { def }}{=}(1 / s) F(s)+D$ is symmetric negative imaginary for any symmetric matrix $D$.

\section{A. Strongly strictly negative imaginary systems}

We now define strongly strictly negative imaginary functions in the same spirit of the definition of SSPR.

Definition 5: Let $G: \mathbb{C} \longrightarrow \mathbb{C}^{m \times m}$ be a real transfer function. Then, $G(s)$ is strongly strictly negative imaginary (SSNI) if for some $\varepsilon>0$, the transfer function $G(s-\varepsilon)$ is NI and $i\left[G(s)-G(-s)^{\top}\right]$ has full normal rank.

Remark 4: The full normal rank condition is essential in the above definition, as this class of systems will be needed for internal stability of positive feedback interconnections of NI and SSNI systems. If we were not to impose the full normal rank condition on the SSNI class, then the feedback interconnection of a NI system and an SSNI system would not be internally stable as demonstrated via the following simple example: Let $P(s)=\left[\begin{array}{ll}1 & 1 \\ 1 & 1\end{array}\right]$ which is clearly NI and let $Q(s)=\frac{1}{s+1}\left[\begin{array}{ll}1 & 1 \\ 1 & 1\end{array}\right]$ which fulfils all properties of SSNI

${ }^{3}$ Notice that the residual matrix $K_{0}$ is the product of the imaginary unit $i$ by the residue at $\omega_{0}$. except for the full normal rank condition. The positive feedback interconnection of $P(s)$ and $Q(s)$ is not internally stable as there exists a closed-loop pole at $s=3$.

Next, we consider an example of NI non-rational transfer function introduced in [20] and show that it is not SSNI. We also introduce an example of a non-rational transfer function that is SSNI

Example 3.1: Let $G(s)=-s\left(e^{-s T}+1\right)$, with $T \in \mathbb{R}_{+}$being a positive delay. As shown in [20], $G(s)$ is negative imaginary. On the other hand $G(s)$ is not SSNI. In fact, let $G_{\varepsilon}(s) \stackrel{\text { def }}{=} G(s-\varepsilon)$ and let $s=\sigma+i \omega$. A direct calculation yields

$i\left[G_{\varepsilon}(s)-G_{\varepsilon}(s)^{*}\right]=2\left[\omega+e^{(\varepsilon-\sigma) T}(\omega \cos (\omega T)-(\sigma-\varepsilon) \sin (\omega T))\right]$ so that, it is immediate to check that for all $\varepsilon>0, i\left[G_{\varepsilon}(s)-G_{\varepsilon}(s)^{*}\right]$ is negative for $s=\varepsilon / 2+j \pi / T$.

Consider now $G(s)=\frac{e^{-s}+4}{s+1}$. To show that $G(s)$ is SSNI, we set $\varepsilon=1 / 2$. Clearly, $G(s)$ is analytic for $\mathfrak{R e}\{s\}>-\varepsilon$, so that $G_{\varepsilon}(s) \stackrel{\text { def }}{=}$ $G(s-\varepsilon)$ is analytic for $\mathfrak{R e}\{s\}>0$. A direct calculation yields

$$
i\left[G_{\varepsilon}(s)-G_{\varepsilon}(s)^{*}\right]=\frac{2[A \omega+B \sin (\omega)]}{|s+1 / 2|^{2}}
$$

where $A \stackrel{\text { def }}{=}\left(4+\sqrt{e} e^{-\sigma} \cos (\omega)\right)>2$ for all positive $\sigma$ and $B \stackrel{\text { def }}{=} \sqrt{e}(\sigma+1 / 2) e^{-\sigma}$ is easily seen to be in the interval $(0,2)$ for all positive $\sigma$. Therefore, it is easy to check that $i\left[G_{\varepsilon}(s)-G_{\varepsilon}(s)^{*}\right]$ satisfies all the prescribed sign conditions so that $G_{\varepsilon}(s)$ is NI and $G(s)$ is SSNI.

Next, we show that SSNI can be checked via conditions on the imaginary axis. To this aim, we need some preliminary results.

Lemma 4: Let $G: \mathbb{C} \longrightarrow \mathbb{C}^{m \times m}$ be a real transfer function. Then, $G(s)$ is SSNI if and only if there exists $\varepsilon>0$ such that

(i) $G(s)$ is analytic in $\{s \in \mathbb{C}: \mathfrak{R e}\{s\}>-\varepsilon\}$;

(ii) $i\left[G(s)-G(s)^{*}\right]>0$ for all $s \in\{s \in \mathbb{C}: \mathfrak{R e}\{s\}>-\varepsilon$, Im $\{s\}>0\}$; (iii) $i\left[G(s)-G(s)^{*}\right]=0$ for all $s \in\{s \in \mathbb{C}: \mathfrak{R e}\{s\}>-\varepsilon, \mathfrak{I m}\{s\}=0\}$; (iv) $i\left[G(s)-G(s)^{*}\right]<0$ for all $s \in\{s \in \mathbb{C}: \mathfrak{R e}\{s\}>-\varepsilon, \mathfrak{I m}\{s\}<0\}$.

Proof: Definition 5 trivially gives equivalence to the existence of $\varepsilon>$ 0 such that conditions (i)-(iv) are satisfied with non-strict inequalities in (ii) and (iv) on $i\left[G(s)-G(s)^{*}\right]$. We hence only need to show that if $G$ is SSNI, then the inequalities in (ii) and (iv) are indeed strict. We prove only that (ii) is strict since (iv) follows by symmetry. Let $G$ be analytic in $\mathbb{C}_{-\varepsilon} \stackrel{\text { def }}{=}\{s \in \mathbb{C}: \mathfrak{R e}\{s\}>-\varepsilon\}$ and assume by contradiction that there exist $s_{0} \in\{s \in \mathbb{C}: \mathfrak{R e}\{s\}>-\varepsilon$ and $\mathfrak{I m}\{s\}>0\}$ and a nonzero vector $v$ such that $v^{*}\left(i\left[G\left(s_{0}\right)-G\left(s_{0}\right)^{*}\right]\right) v=0$. Let $\varepsilon_{1}<\varepsilon$ be such that $\mathfrak{R e}\left\{s_{0}\right\}>-\varepsilon_{1}$. Since $G$ is analytic in $\mathbb{C}_{-\varepsilon}, v^{*}(i[G(s)-$ $\left.\left.G(s)^{*}\right]\right) v$ is harmonic in the same domain so that, by considering an arbitrarily large real number $M$ and the compact set $\mathscr{C} \stackrel{\text { def }}{=}\{s \in \mathbb{C}: M \geq$ $\mathfrak{R e}\{s\} \geq-\varepsilon_{1}$ and $\left.M \geq \mathfrak{I m}\{s\} \geq 0\right\} \subset \mathbb{C}_{-\varepsilon}$, if $v^{*}\left(i\left[G(s)-G(s)^{*}\right]\right) v$ restricted to $\mathscr{C}$ attains its minimum at a point $s_{0}$ in the interior of $\mathscr{C}$, then $v^{*}\left(i\left[G(s)-G(s)^{*}\right]\right) v$ is constant. Clearly, $v^{*}\left(i\left[G(s)-G(s)^{*}\right]\right) v \geq$ 0 for all $s \in \mathscr{C}$ and, by taking $M$ sufficiently large, $s_{0}$ is in the interior of $\mathscr{C}$ so that $v^{*}\left(i\left[G(s)-G(s)^{*}\right]\right) v$ is constantly equal to 0 . This is a contradiction, since Definition 5 requires that $i\left[G(s)-G(-s)^{\top}\right]$ has full normal rank.

Lemma 5: Let $g: \mathbb{C} \longrightarrow \mathbb{C}$ be a scalar, real, rational, strictly proper transfer function. Assume that $g(s)$ is a NI function. Then, the relative degree of $g(s)$ is at most 2 and all the finite zeros of $g(s)$ are in the closed left half-plane. Moreover, if $i\left[g(i \omega)-g(i \omega)^{*}\right]>0$ for all $\omega \in(0, \infty)$, then all the finite zeros of $g(s)$ are in the open left half-plane.

Proof: As a consequence of [20, Theorem 3.1] we have that $f(s) \stackrel{\text { def }}{=}$ $s g(s)$ is PR. Then, the relative degree of $f(s)$ is at most 1 and all the finite zeros of $f(s)$ are in the closed left half-plane. Therefore, the relative degree of $g(s)$ is at most 2 and all the finite zeros of $g(s)$ 
are in the closed left half-plane. Moreover, if $i\left[g(i \omega)-g(i \omega)^{*}\right]>0$, the only point of the imaginary axis in which $g$ could vanish is 0 . If, however, $g(0)=0$ then $f(s)$ would have a double zero at the origin which is in contrast with positive realness.

Remark 5: Note that the strictly proper assumption in Lemma 5 is essential to this observation. Indeed, it is possible to have bi-proper transfer functions such as $g(s)=\frac{1-s}{1+s}$ that have zeros in the open right half-plane and are still SSNI and hence also NI. This is a crucial difference between PR functions (that are necessarily minimum phase) and NI functions.

Lemma 6: Let $g: \mathbb{C} \longrightarrow \mathbb{C}$ be a scalar, real, rational, proper transfer function. Assume that $g(s)$ is a SSNI function. If $g(0)=0$, then the multiplicity of the zero in the origin of $g(s)$ is equal to 1 .

Proof: Since $g(s)$ is a SSNI function, it has no poles in zero and we can expand $g(s)$ at the origin as

$$
g(s)=\sum_{k=h}^{\infty} r_{k} s^{k},
$$

where $h$ is the multiplicity of the zero at the origin of $g$. Let $s=\varepsilon e^{i \theta}$ $0<\theta<\pi$. If $\varepsilon$ is sufficiently small, $i\left[g(s)-g(s)^{*}\right]$ has the same sign of $-2 r_{h} \varepsilon^{h} \sin (h \theta)$, so that it can be positive for any $\theta \in(0, \pi)$ only if $h=1$.

We now present necessary and sufficient conditions on the imaginary axis for a system to be SSNI.

Theorem 3: Let $G: \mathbb{C} \longrightarrow \mathbb{C}^{m \times m}$ be a real, rational, proper, symmetric transfer function. Then $G(s)$ is SSNI if and only if (i) $G(s)$ has all its poles in $\{s \in \mathbb{C}: \mathfrak{R e}\{s\}<0\}$;

(ii) $i\left[G(i \omega)-G(i \omega)^{*}\right]>0$ for all $\omega \in(0, \infty)$;

(iii) there exist $\sigma_{0}>0$ and $\delta>0$ such that

$$
\underline{\sigma}\left[\omega^{3} i\left[G(i \omega)-G(i \omega)^{*}\right]\right]>\sigma_{0} \quad \forall \omega \geq \delta ;
$$

(iv)

$$
Q \stackrel{\text { def }}{=} \lim _{\omega \rightarrow 0^{+}}(1 / \omega) i\left[G(i \omega)-G(i \omega)^{*}\right]>0 .
$$

Proof: Necessity of (i) and (ii) is trivial from Lemma 4. We now show necessity of condition (iii). Essentially, we need to show that for any vector $v$ the relative degree of $i\left[g^{\prime}(i \omega)-g^{\prime}(i \omega)^{*}\right]$, where $g^{\prime}(s) \stackrel{\text { def }}{=} v^{\top} G(s) v$, is at most 3. Assume by contradiction that this is not the case so that $g(s) \stackrel{\text { def }}{=} g^{\prime}(s)-g^{\prime}(\infty)$ is a rational strictly proper SSNI function such that $i\left[g(i \omega)-g(i \omega)^{*}\right]$ tends to zero, as $\omega \rightarrow \infty$, faster than $1 / \omega^{3}$. Then, it is easy to check that the relative degree of $g$ is at least 2 and, in view of Lemma 5, the relative degree of $g$ is exactly 2 . In view of Lemma 5 we can write $g(s)$ as

$$
g(s)=K \frac{n(s)}{d(s)}=K \frac{s^{n-2}+a_{n-3} s^{n-3}+\cdots+a_{0}}{s^{n}+b_{n-1} s^{n-1}+\cdots+b_{0}},
$$

with $a_{i}$ and $b_{i}$ strictly positive. By imposing that $i\left[g(i \omega)-g(i \omega)^{*}\right]$ tends to zero, as $\omega \rightarrow \infty$, faster than $1 / \omega^{3}$, we get that $n \geq 3$ and $a_{n-3}=b_{n-1}$. Now, we can compute

$$
i\left[g(i \omega-\varepsilon)-g(i \omega-\varepsilon)^{*}\right]=\frac{-4 K \varepsilon \omega}{|d(i \omega-\varepsilon)|^{2}}\left[\left(\varepsilon^{2}+\omega^{2}\right)^{n-2}+T_{2 n-6}\right]
$$

with $T_{2 n-6}$ being a polynomial in $\omega$ of degree equal to $2 n-6$. Therefore for a sufficiently large $\omega, i\left[g(i \omega)-g(i \omega)^{*}\right]$ is negative for any positive $\varepsilon$.

We now show necessity of condition (iv). Assume that $G$ is SSNI. Then clearly the limit $Q$ defined in (4) exists and is positive semidefinite. Assume by contradiction that $Q$ is singular and let $v \in \operatorname{ker} Q$. Let $g^{\prime}(s) \stackrel{\text { def }}{=} v^{\top} G(s) v$. Clearly, $g^{\prime}(s)$ is a rational proper SSNI function and $g(s) \stackrel{\text { def }}{=} g^{\prime}(s)-g^{\prime}(\infty)$ is a rational strictly proper SSNI function such that

$$
\lim _{\omega \rightarrow 0}(1 / \omega) i\left[g(i \omega)-g(i \omega)^{*}\right]=0 .
$$

In view of Lemma 5 we can write $g(s)$ as

$$
g(s)=K \frac{n(s)}{d(s)}=K \frac{1+a_{1} s+a_{2} s^{2}+\cdots+a_{m} s^{m}}{1+b_{1} s+b_{2} s^{2}+\cdots+b_{n} s^{n}}, \quad m<n
$$

with $a_{i}$ and $b_{i}$ strictly positive. Then (5) implies $a_{1}=b_{1}$. Now

$$
g(s)-K=K \frac{n(s)-d(s)}{d(s)}
$$

is SSNI as well, so that the multiplicity of its zero in the origin is at most equal to 1 . Therefore $a_{1} \neq b_{1}$.

As for sufficiency, assume that $G(s)$ is real symmetric and rational and that it satisfies $(i),(i i),(i i i)$ and (iv). We now show that we can choose $\varepsilon>0$ in such a way that

$$
i\left[G(-\varepsilon+i \omega)-G(-\varepsilon+i \omega)^{*}\right]>0, \forall \omega \in(0, \infty) .
$$

In view of condition (ii), we have that for all $\omega_{2}>\omega_{1}>0$, there exists $\varepsilon>0$ such that

$$
i\left[G(-\varepsilon+i \omega)-G(-\varepsilon+i \omega)^{*}\right]>0, \forall \omega \in\left[\omega_{1}, \omega_{2}\right],
$$

so that it is sufficient to show that given an arbitrarily small $\omega_{1}$ and an arbitrarily large $\omega_{2}$, there exists $\varepsilon>0$ such that

$$
i\left[G(-\varepsilon+i \omega)-G(-\varepsilon+i \omega)^{*}\right]>0, \forall \omega \in\left(0, \omega_{1}\right)
$$

and

$$
i\left[G(-\varepsilon+i \omega)-G(-\varepsilon+i \omega)^{*}\right]>0, \forall \omega \in\left(\omega_{2}, \infty\right) .
$$

As for (8), let $\delta \stackrel{\text { def }}{=} i \omega-\varepsilon$ and consider the following expansion of $G(\delta)$ :

$$
G(\delta)=D_{0}+\delta D_{1}+\delta^{2} D_{2}+\ldots
$$

which clearly converges for $\delta$ sufficiently small (if we considered a minimal realization $G(s)=C(s I-A)^{-1} B+D$, we would have $D_{0} \stackrel{\text { def }}{=} D-C A^{-1} B$ and $D_{i} \stackrel{\text { def }}{=}-C A^{-i-1} B$, for $\left.i>1\right)$. Since $G(s)$ is real symmetric by standing assumption, $D_{i}=D_{i}^{\top}$. Moreover, $Q \stackrel{\text { def }}{=}$ $\lim _{\omega \rightarrow 0^{+}}(1 / \omega) i\left[G(i \omega)-G(i \omega)^{*}\right]=-2 D_{1}$, so that by assumption (iv), we have $D_{1}<0$. Now a direct calculation gives

$$
i\left[G(-\varepsilon+i \omega)-G(-\varepsilon+i \omega)^{*}\right]=-\omega 2 D_{1}+i \sum_{j=2}^{\infty}\left[\delta^{j}-\left(\delta^{*}\right)^{j}\right] D_{j} .
$$

Now we observe that

$$
i \sum_{j=2}^{\infty}\left[\delta^{j}-\left(\delta^{*}\right)^{j}\right] D_{j}=-2 \omega \sum_{j=2}^{\infty} \sum_{k=0}^{j-1}\left[\delta^{k}\left(\delta^{*}\right)^{j-1-k}\right] D_{j},
$$

so that

$$
\begin{aligned}
\left\|i \sum_{j=3}^{\infty}\left[\delta^{j}-\left(\delta^{*}\right)^{j}\right] D_{j}\right\| & \leq 2 \omega \sum_{j=2}^{\infty} j \varepsilon^{j-1}\left\|D_{j}\right\| \\
& =2 \omega \varepsilon \sum_{j=2}^{\infty} j \varepsilon^{j-2}\left\|D_{j}\right\| \leq 2 \omega \sigma \varepsilon
\end{aligned}
$$

for a certain $\sigma$ which does not increase as $\varepsilon$ tends to zero. Since, by choosing a sufficiently small $\varepsilon$ we can make $-D_{1}>\sigma \varepsilon I$, we have (8). Now we prove (9). Let $G(s)=C(s I-A)^{-1} B+D$ be a a minimal realization so that $G(-\varepsilon+i \omega)=G(i \omega)+\varepsilon \Delta(i \omega)$, with $\Delta(s) \stackrel{\text { def }}{=} C(s I-A)^{-1}(s I-\varepsilon I-A)^{-1} B$. We expand $\Delta$ around infinity as

$$
\Delta(i \omega)=\frac{C B}{(i \omega)^{2}}+\frac{\Delta_{3}}{(i \omega)^{3}}+\frac{\Delta_{r}(i \omega)}{(i \omega)^{4}}
$$

where $\Delta_{3}$ remains bounded as $\varepsilon$ tends to zero and $\Delta_{r}(i \omega)$ remains bounded as $\varepsilon$ tends to zero and $\omega$ tends to $+\infty$. Then, we have

$$
\begin{aligned}
& i\left[G(-\varepsilon+i \omega)-G(-\varepsilon+i \omega)^{*}\right]= \\
& \quad=i\left[G(i \omega)-G(i \omega)^{*}\right]+\frac{\varepsilon\left(-\Delta_{3}-\Delta_{3}^{\top}\right)}{\omega^{3}}+\frac{i}{\omega^{4}}\left[\Delta_{r}(i \omega)-\Delta_{r}(i \omega)^{*}\right]
\end{aligned}
$$


so that, in view of condition (iii), (9) holds.

Now we can apply Lemma 3 to the function $G(s-\varepsilon)$ and we immediately see that it is NI so that $G$ is SSNI

Remark 6: In view of the symmetry, we have the following expansion at infinity: $i\left[G(i \omega)-G(i \omega)^{*}\right]=(1 / \omega) P_{1}+\left(1 / \omega^{3}\right) P_{3}+\ldots$, so that it is easy to see that condition (3) may be equivalently rewritten, in the same spirit of the condition obtained in [23], as

$$
\lim _{|\omega| \rightarrow \infty} \omega^{m+2 \rho} \operatorname{det}\left(i\left[G(i \omega)-G(i \omega)^{*}\right]\right)>0
$$

where $\rho$ is the nullity of $P_{1}=\lim _{|\omega| \rightarrow \infty} i \omega\left[G(i \omega)-G(i \omega)^{*}\right]$.

In the scalar case, condition (4) has an intuitive interpretation as a departure gradient on the phase of the frequency response (see Lemma 3.7 in [25] for details).

The following result is the counterpart of Theorem 2 for SSPR and SSNI transfer functions.

Theorem 4: Let $F: \mathbb{C} \longrightarrow \mathbb{C}^{m \times m}$ be a real, rational, symmetric SSPR transfer function. Then, there exists $\varepsilon>0$ such that $G(s) \stackrel{\text { def }}{=} \frac{F(s)}{s+\varepsilon}+D$ is SSNI for any symmetric matrix $D$. Conversely, let $G: \mathbb{C} \longrightarrow \mathbb{C}^{m \times m}$ be a real, rational, proper, symmetric SSNI transfer function. Then, there exists $\varepsilon>0$ such that $(s+\varepsilon)(G(s)-G(\infty))$ is SSPR.

Proof: We start proving the first statement. Since $F(s)$ is SSPR, a value $\varepsilon>0$ exists such that $F(s-\varepsilon)$ is PR. Then, by Theorem 2 it is found that $\hat{G}(s) \stackrel{\text { def }}{=} \frac{F(s-\varepsilon)}{s}$ is NI. On the other hand, this implies that $G(s)=\frac{F(s)}{s+\varepsilon}$ is SSNI.

We prove the second statement. Since $G(s)$ is SSNI, there exists $\varepsilon>0$ such that $G(s-\varepsilon)$ is NI. Thus, by Theorem 2 we find that $s[G(s-\varepsilon)-G(\infty)]$ is PR. This in turn implies that $(s+$ $\varepsilon)[G(s)-G(\infty)]$ is SSPR.

\section{B. Weakly strictly negative imaginary systems}

Reference [26] and earlier results use a weaker definition of strictly negative imaginary systems to obtain robust stability results. This weaker notion imposes only conditions (i) and (ii) of Theorem 3. In light of this, we introduce the following definition.

Definition 6: The real, rational, proper, symmetric transfer function $G: \mathbb{C} \longrightarrow \mathbb{C}^{m \times m}$ is weakly strictly negative imaginary (WSNI) if it satisfies conditions (i) and (ii) of Theorem 3.

Equation (16) in [11] gives a MIMO example of a SSNI system, which is hence also a MIMO example of a WSNI system.

Notice that this concept of WSNI is only defined for the rational case. It coincides, in the symmetric case, to the concept of "strictly negative imaginary system" used in [27]. The following two examples show that conditions (iii) and (iv) in Theorem 3 are not implied by the first two, i.e., the notion of WSNI is indeed a weaker notion than that of SSNI.

Example 3.2: Consider the transfer function

$$
G(s)=\frac{2 s+1}{(s+1)^{2}} .
$$

It is easily seen that $G(s)$ is NI. A simple calculation shows that

$$
i\left[G(i \omega-\varepsilon)-G(i \omega-\varepsilon)^{*}\right]=\frac{4 \omega}{\left[\omega^{2}+(1-\varepsilon)^{2}\right]^{2}}\left(\omega^{2}-\varepsilon+\varepsilon^{2}\right),
$$

which proves that conditions (i), (ii) and (iii) in Theorem 3 are satisfied; in particular, this means that $G(s)$ is WSNI. However, it is not SSNI, because in this case (4) yields $Q=\lim _{\omega \rightarrow 0^{+}} \frac{4 \omega^{2}}{\left(\omega^{2}+1\right)^{2}}=0$. This result is consistent with Definition 5. Indeed, for any $\varepsilon>0$, there always exists a sufficiently small $\omega>0$ such that the numerator in (10) is negative.
Example 3.3: Consider the transfer function

$$
G(s)=\frac{s+3}{(s+1)^{3}} .
$$

Again, $G(s)$ is NI, and in this case

$i\left[G(i \omega-\varepsilon)-G(i \omega-\varepsilon)^{*}\right]=\frac{4 \omega\left[4+6 \varepsilon^{2}-\varepsilon^{3}-\varepsilon\left(\omega^{2}+9\right)\right]}{\left(1+\varepsilon^{2}-2 \varepsilon+\omega^{2}\right)^{3}}$.

Thus, conditions (i), (ii) in Theorem 3 are satisfied, which means that $G(s)$ is WSNI. Condition (iv) in Theorem 3 is also satisfied, since in this case (4) gives $Q=\lim _{\omega \rightarrow 0^{+}} \frac{16}{\left(\omega^{2}+1\right)^{3}}=16>0$. However, $G(s)$ is not SSNI because (iii) in Theorem 3 is not satisfied. Again, this result is consistent with Definition 5, since for any $\varepsilon>0$, there always exists a sufficiently large $\omega>0$ such that the numerator in (11) becomes negative.

The next theorem shows that the definition of WSNI corresponds to a sign property on the closed right-half plane.

Theorem 5: Let $G: \mathbb{C} \longrightarrow \mathbb{C}^{m \times m}$ be a real, rational, proper transfer function. Then, $G(s)$ is WSNI if and only if there exists $\varepsilon>0$ such that

(i) $G(s)$ is analytic in $\{s \in \mathbb{C}: \mathfrak{R e}\{s\}>-\varepsilon\}$;

(ii) $i\left[G(s)-G(s)^{*}\right]>0$ for all $s \in\{s \in \mathbb{C}: \mathfrak{R e}\{s\} \geq 0, \quad \operatorname{Im}\{s\}>0\}$; (iii) $i\left[G(s)-G(s)^{*}\right]=0$ for all $s \in\{s \in \mathbb{C}: \mathfrak{R e}\{s\} \geq 0$, Im $\{s\}=0\}$; (iv) $i\left[G(s)-G(s)^{*}\right]<0$ for all $s \in\{s \in \mathbb{C}: \mathfrak{R e}\{s\} \geq 0$, $\operatorname{Im}\{s\}<0\}$.

Proof: Sufficiency is trivial by restricting on the imaginary axis. Necessity can be proven as follows: if $G$ is WSNI, then $(i)$ is satisfied and $G$ is NI (from Lemma 3). Moreover, if $G$ is NI, then (ii)-(iv) in Definition 4 are satisfied. Appending the imaginary axis properties of $G$ to the conditions (ii)-(iv) in Definition 4 (since $G$ is WSNI) yields (ii)-(iv) since $G$ fulfils (i).

\section{Interconnections of negative imaginary systems}

The following result shows under what circumstances are NI, WSNI and SSNI properties preserved when such systems are interconnected in feedback.

Theorem 6: Let $S_{1}: \mathbb{C} \rightarrow \mathbb{C}^{m_{1} \times m_{1}}$ be NI (resp. WSNI or SSNI) and $S_{2}: \mathbb{C} \rightarrow \mathbb{C}^{m_{2} \times m_{2}}$ be NI (resp. WSNI or SSNI). Let $0<a, b \leq$ $\min \left\{m_{1}, m_{2}\right\}$ and suppose the feedback interconnection corresponding to the Redheffer Star product $S_{1} \star S_{2}$ be internally stable. ${ }^{4}$ Then $S_{1} \star S_{2}$ is NI (resp. WSNI or SSNI).

Furthermore, if

- $a=b=m_{2}<m_{1}$, then $S_{1} \star S_{2}=F_{l}\left(S_{1}, S_{2}\right)$;

- $a=b=m_{1}<m_{2}$, then $S_{1} \star S_{2}=F_{u}\left(S_{2}, S_{1}\right)$; $\begin{aligned}- & a=b=m_{2}=m_{1} / 2, S_{1}=\left[\begin{array}{cc}P & I_{a} \\ I_{a} & 0\end{array}\right] \text { and } S_{2}=Q \text {, then } S_{1} \star S_{2}= \\ & P+Q\end{aligned}$

- $a=b=m_{2}=m_{1} / 2, S_{1}=\left[\begin{array}{cc}0 & I_{a} \\ I_{a} & P\end{array}\right]$ and $S_{2}=Q$, then $S_{1} \star S_{2}=$ $Q\left(I_{a}-P Q\right)^{-1}$

- $2 a=2 b=m_{1}=m_{2}, S_{1}=\left[\begin{array}{cc}0 & I_{a} \\ I_{a} & P\end{array}\right]$ and $S_{2}=\left[\begin{array}{cc}Q & I_{a} \\ I_{a} & 0\end{array}\right]$, then $S_{1}$ * $S_{2}=\left[\begin{array}{cc}-P & I_{a} \\ I_{a} & -Q\end{array}\right]^{-1}=\left[\begin{array}{cc}Q\left(I_{a}-P Q\right)^{-1} & \left(I_{a}-Q P\right)^{-1} \\ \left(I_{a}-P Q\right)^{-1} & P\left(I_{a}-Q P\right)^{-1}\end{array}\right]$ which corresponds to the positive feedback interconnection $[P, Q]$.

Proof: Given $S_{1}(s), S_{2}(s)$ and complex vectors $y_{1}, y_{2}, u_{1}, u_{2}, \alpha, \beta$ of compatible dimension satisfying $\left[\begin{array}{c}y_{1} \\ \alpha\end{array}\right]=S_{1}(s)\left[\begin{array}{l}u_{1} \\ \beta\end{array}\right]$ and $\left[\begin{array}{l}\beta \\ y_{2}\end{array}\right]=$

${ }^{4}$ This is the standard meaning of "internal stability", i.e. add two extra exogenous input signals to the internal signals and ensure that all output signals and all internal signals are energy-bounded for any energy-bounded exogenous input excitation. 


$$
\begin{aligned}
& S_{2}(s)\left[\begin{array}{l}
\alpha \\
u_{2}
\end{array}\right] \text {, it follows that }\left[\begin{array}{l}
y_{1} \\
y_{2}
\end{array}\right]=S_{1}(s) \star S_{2}(s)\left[\begin{array}{l}
u_{1} \\
u_{2}
\end{array}\right] \text {. Then, for all } \\
& {\left[\begin{array}{c}
u_{1} \\
\beta
\end{array}\right] \in \mathbb{C}^{m_{1}},\left[\begin{array}{c}
\alpha \\
u_{2}
\end{array}\right] \in \mathbb{C}^{m_{2}}:}
\end{aligned}
$$

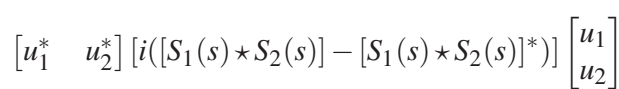

$$
\begin{aligned}
& =i\left[\begin{array}{ll}
u_{1}^{*} & u_{2}^{*}
\end{array}\right]\left[\begin{array}{l}
y_{1} \\
y_{2}
\end{array}\right]-i\left[\begin{array}{ll}
y_{1}^{*} & y_{2}^{*}
\end{array}\right]\left[\begin{array}{l}
u_{1} \\
u_{2}
\end{array}\right] \\
& =i\left[\begin{array}{ll}
u_{1}^{*} & \beta^{*}
\end{array}\right]\left[\begin{array}{c}
y_{1} \\
\alpha
\end{array}\right]-i\left[\begin{array}{ll}
y_{1}^{*} & \alpha^{*}
\end{array}\right]\left[\begin{array}{c}
u_{1} \\
\beta
\end{array}\right] \\
& +i\left[\begin{array}{ll}
\alpha^{*} & u_{2}^{*}
\end{array}\right]\left[\begin{array}{c}
\beta \\
y_{2}
\end{array}\right]-i\left[\begin{array}{ll}
\beta^{*} & y_{2}^{*}
\end{array}\right]\left[\begin{array}{c}
\alpha \\
u_{2}
\end{array}\right] \\
& =\left[\begin{array}{ll}
u_{1}^{*} & \beta^{*}
\end{array}\right]\left[i\left(S_{1}(s)-S_{1}(s)^{*}\right)\right]\left[\begin{array}{c}
u_{1} \\
\beta
\end{array}\right] \\
& +\left[\begin{array}{ll}
\alpha^{*} & u_{2}^{*}
\end{array}\right]\left[i\left(S_{2}(s)-S_{2}(s)^{*}\right)\right]\left[\begin{array}{c}
\alpha \\
u_{2}
\end{array}\right] .
\end{aligned}
$$

Since the Redheffer star interconnection is internally stable, the three respective results (NI, WSNI, SSNI) then follow by applying Definition 4, Theorem 5 or Lemma 4 respectively on the corresponding domains of $s \in \mathbb{C}$ for $S_{1}(s)$ and $S_{2}(s)$.

The five cases where $a, b, S_{1}$ and $S_{2}$ are restricted are trivial consequences of a Redheffer calculation.

Notice, that this result holds for the general — possibly nonrational - case.

Example 3.4: This example shows that it is not possible to mix and match properties of $S_{1}$ and $S_{2}$ for the strict results in Theorem 6

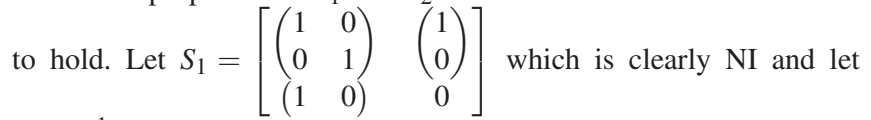
$S_{2}=\frac{1}{s+1}$ which is SSNI (and also WSNI and hence NI). Then $S_{1}$ * $S_{2}=\left[\begin{array}{cc}1+\frac{1}{s+1} & 0 \\ 0 & 1\end{array}\right]$ which is only NI (and not WSNI nor SSNI).

\section{CONCLUDING REMARKS}

We have drawn a full picture which illustrates the various grades of strictly negative imaginary systems and the relationships that exist between the notions of positive real and negative imaginary systems. The approach followed in this paper hinges entirely on properties of the transfer function matrix and is founded on general definitions that do not require the transfer functions to be rational.

\section{REFERENCES}

[1] O. Brune, The synthesis of a finite two-terminal network whose drivingpoint impedance is a prescribed function of frequency. Journal of Mathematical Physics, 10: 191-236, 1931.

[2] B.D.O. Anderson, and S. Vongpanitlerd. Network Analysis and Synthesis: a Modern Systems Theory Approach. Upper Saddle River, NJ: Prentice Hall, 1973.

[3] B. Brogliato, R. Lozano, B. Maschke and O. Egeland. Dissipative Systems Analysis and Control Theory and Applications. 2nd ed. New York: Springer-Verlag, 2007.

[4] A. Lanzon, and I.R. Petersen. Stability robustness of a feedback interconnection of systems with negative imaginary frequency response. IEEE Transactions on Automatic Control, 53(4):1042-1046, 2008.

[5] J. Xiong, I.R. Petersen, and A. Lanzon. A negative imaginary lemma and the stability of interconnections of linear negative imaginary systems. IEEE Transactions on Automatic Control, 55(10):2342-2347, 2010.

[6] M.A. Mabrok, A. Kallapur, I.R. Petersen and A. Lanzon. Generalizing negative imaginary systems theory to include free body dynamics: Control of highly resonant structures with free body motion. IEEE Transactions on Automatic Control, 59(10):2692-2707, Oct 2014.
[7] I.R. Petersen, and A. Lanzon. Feedback control of negative-imaginary systems. IEEE Control Systems Magazine, 30(5):54-72, 2010.

[8] B. Bhikkaji, S. O. R. Moheimani, and I. R. Petersen. A negative imaginary approach to modeling and control of a collocated structure. IEEE/ASME Transactions on Mechatronics, 17(4):717-727, 2012.

[9] B. Bhikkaji, and S. Moheimani. Fast scanning using piezoelectric tube nanopositioners: A negative imaginary approach. Proc. IEEE/ASME Int. Conf. Advanced Intelligent Mechatronics AIM, Singapore, pp. 274 279, July 2009.

[10] I. A. Mahmood, S. O. R. Moheimani, and B. Bhikkaji. A new scanning method for fast atomic force microscopy. IEEE Transactions on Nanotechnology, 10(2):203-216, 2011.

[11] M.A. Mabrok, A. Kallapur, I.R. Petersen and A. Lanzon. Spectral conditions for negative imaginary systems with applications to nanopositioning. IEEE/ASME Transactions on Mechatronics, 19(3):895903, 2014.

[12] C. Cai, and G. Hagen. Stability analysis for a string of coupled stable subsystems with negative imaginary frequency response. IEEE Transactions on Automatic Control, 55(8):1958-1963, 2010.

[13] J. Wang, A. Lanzon, and I.R. Petersen. Robust output feedback consensus for networked negative-imaginary systems. IEEE Transactions on Automatic Control, 60(9):2547-2552, 2015.

[14] J. Xiong, I.R. Petersen, and A. Lanzon. On lossless negative imaginary systems. Automatica, 48(6): 1213-1217, 2012.

[15] M. Opmeer. Infinite-dimensional negative imaginary systems. IEEE Transactions on Automatic Control, 56(12):2973-2976, 2011.

[16] A. van der Schaft. Positive feedback interconnection of Hamiltonian systems. Proceedings of the 50th IEEE Conference on Decision and Control and European Control Conference, Orlando, FL, USA, Dec 2011.

[17] J. Xiong, A. Lanzon, and I. R. Petersen. Negative imaginary lemmas for descriptor systems. IEEE Transactions on Automatic Control, In press.

[18] S. Patra, and A. Lanzon. Stability analysis of interconnected systems with 'mixed' negative-imaginary and small-gain properties. IEEE Transactions on Automatic Control, 56(6):1395-1400, 2011.

[19] M.H. Moghaddam, and H. Khaloozadeh. Comments on "A Note on Spectral Conditions for Positive Realness of Single-InputSingle-Output Systems". IEEE Transactions on Automatic Control, 58(4):1091-1092, 2013.

[20] A. Ferrante, and L. Ntogramatzidis, "Some new results in the theory of negative imaginary systems with symmetric transfer matrix function". Automatica, 49(7): 2138-2144, 2013.

[21] H.K. Khalil. Nonlinear Systems. 3rd ed. New Jersey: Prentice Hall, 2002.

[22] J. T. Wen. Time domain and frequency domain conditions for strict positive realness. IEEE Transactions on Automatic Control, vol.33(10):988-992, 1988.

[23] M. Corless, and R. Shorten. On the Characterization of Strict Positive Realness for General Matrix Transfer Functions. IEEE Transactions on Automatic Control, 55(8):1899-1904, 2010.

[24] M. Hakimi-Moghaddam, and H. Khaloozadeh. "Characterization of strictly positive real multivariable systems". IMA Journal of Mathematical Control and Information. DOI: 10.1093/imamci/dnt040, 2013.

[25] A. Lanzon, Z. Song, S. Patra, and I.R. Petersen. A strongly strict negative-imaginary lemma for non-minimal linear systems Communications in Information and Systems, 11(2):139-152, 2011.

[26] Z. Song, A. Lanzon, S Patra, I.R. Petersen. A negative-imaginary lemma without minimality assumptions and robust state-feedback synthesis for uncertain negative-imaginary systems Systems and Control Letters, 61(12):1269-1276, 2012.

[27] N. Bajcinca, and M. Voigt. Spectral conditions for symmetric positive real and negative imaginary systems. European Control Conference 2013, pp. 809-814, July 2013. 